\title{
Severe Corneal Morphological Alterations after Excimer Laser Surface Ablation for a High Astigmatism
}

\author{
Anna M. Roszkowska Giovanni W. Oliverio Giuseppe A. Signorino \\ Mario Urso Pasquale Aragona \\ Ophthalmology Unit, Department of Biomedical Sciences, University Hospital of Messina, \\ Messina, Italy
}

\section{Keywords}

Corneal alterations $\cdot$ Excimer laser $\cdot$ Refractive surgery complications

\begin{abstract}
We report long-term alterations of anterior corneal stroma after excimer laser surface ablation for a high astigmatism. The patient claimed progressive visual loss in his right eye (RE) during the last 3 years after bilateral laser-assisted subepithelial keratectomy (LASEK) surgery. His examination comprised visual acuity (UDVA and CDVA), slit-lamp examination, corneal topography and tomography, AS-OCT, and confocal microscopy. The UDVA was 0.1 in his RE and 1.0 in the left eye. The CDVA in the RE was 0.8. The slit-lamp examination showed a stromal lesion in the inferior paracentral corneal zone, with multiple vertical tissue bridges and severe thinning. Corneal topography and tomography showed central flattening with inferior steepening and severe alteration in elevation maps. AS-OCT showed void areas in the anterior stroma with thinning of the underlying tissue, and confocal images were not specific. In this case, progressive corneal steepening and thinning that manifest topographically as inferior ectasia occurred in correspondence to the singular stromal alterations after LASEK.
\end{abstract}

\section{Introduction}

Post-refractive surgery corneal ectasia is defined as progressive steepening and thinning of the cornea, occurring after excimer laser procedures, and it was described first by Seiler in 1998 in relation to laser-assisted in situ keratomileusis [1]. After this report, due to the diffusion of refractive surgery, many ophthalmologists attempted to identify the risk factors and prevention methods to avoid such event that was rapidly recognized as a very severe complication of this technique, like after surface ablation. Since post-laser-assisted in situ 
keratomileusis ectasia occurs with higher prevalence like after surface ablation, photorefractive keratectomy (PRK), or laser-assisted subepithelial keratectomy (LASEK), the major risk factors have been identified exclusively for the former procedure. In fact, the less invasive surface ablation is generally considered as a safer approach that could be recommended occasionally in some so called suspected corneas $[2,3]$. Thus, the post-PRK/LASEK ectasia appears as a sporadic event, and it was rarely reported in the scientific literature confirming a low incidence, with a prevalence of about $0.03 \%$ [2-6].

In this report, we describe an atypical case of corneal complication that occurred 3 years after uneventful excimer laser surface ablation for mixed astigmatism. The consent to publish the clinical data and instrumental images was obtained from the patient, and the IRB approval was obtained.

\section{Case Presentation}

A thirty-seven-year-old man presented to our cornea and refractive surgery center with diagnosis of secondary corneal ectasia in his right eye (RE) after bilateral excimer laser surface ablation with LASEK surgery. The patient reported frequent eye rubbing with foreign body sensation and partial lid closure during sleep in the early postoperative period. He claimed slow progressive visual change in the RE for the last 3 years. When referred to our clinic, his refraction

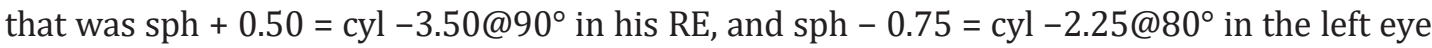
(LE). Preoperative corneal topography showed against-the-rule astigmatism in both eyes with a central thickness of $590 \mu \mathrm{m}$ in the RE and $585 \mu \mathrm{m}$ in the LE (shown in Fig. 1).

We performed complete ophthalmological examination with visual acuity assessment (UDVA and CDVA), refraction, slit-lamp examination, and ophthalmoscopy. The instrumental investigation composed of corneal topography (Antares ${ }^{\circledR}$, CSO - Italy), corneal tomography (Pentcam ${ }^{\circledR}$, Oculus-Germany), anterior segment optical coherence tomography AS-OCT (DRI OCT Triton ${ }^{\circledR}$ Topcon-Japan), and confocal microscopy (Confoscan $4^{\circledR}$, Nidek-Italy).

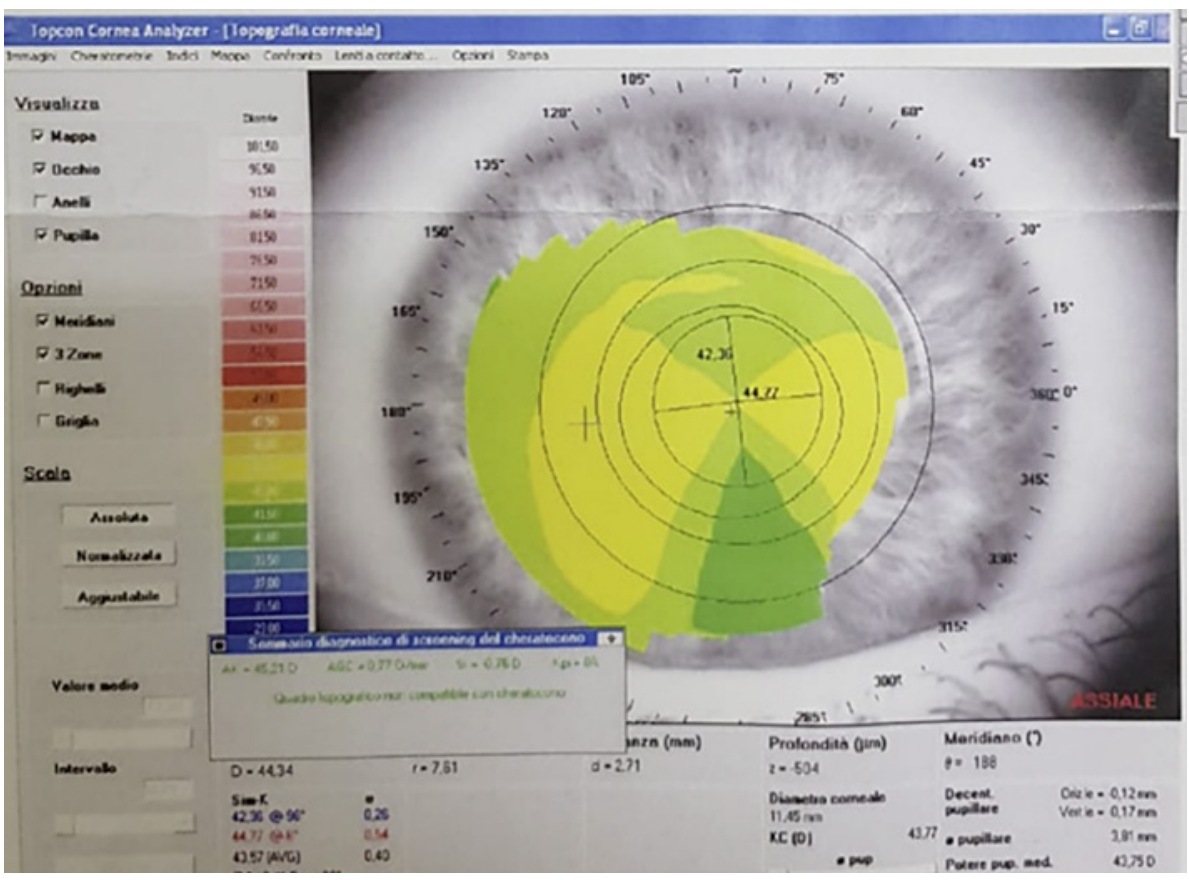

Fig. 1. Preoperative corneal topography exhibited by the patient. 
The UDVA was 0.1 in the RE, with the CDVA 0.8 with sphere $-1=$ cyl $-5 @ 90^{\circ}$. The UDVA in the LE was 1.0.

At the slit-lamp examination, we observed the stromal lesion that was characterized by vertical stromal tissue bridges with thinning and steepening that extended between 5 and 8 o'clock. It appeared to be located likely outside of the treatment zone at $3 \mathrm{mms}$ from the limbus (shown in Fig. 2a). AS-OCT showed important thinning with quite absence of the anterior stroma in the correspondence of the lesion, while the covering epithelium was slightly thickened and measured $82 \mu \mathrm{m}$. The stromal alterations presented numerous void areas that appeared as tissue-lacking zones with irregular stromal debris and hyper-reflective underlying line. The underlying stroma showed severe thinning up to $184 \mu \mathrm{m}$ (shown in Fig. $2 \mathrm{~b}, \mathrm{c}$ ). Corneal topography and tomography showed central flattening and inferior steepening that worsen during last 3 years with against-the-rule high astigmatism up to 15 diopters. The maps are comparable to pellucid ectasia, but the thickness measure resulted to be in the normal range with this device. Posterior elevation map resulted to be highly altered. Confocal images were not specific in the center and not executable in the altered zone. The patient gave written informed consent to publish its case, including publication of images.

\section{Discussion/Conclusion}

In this report, we describe singular corneal changes occurred unilaterally after bilateral uneventful surface correction for a high astigmatism. Corneal ectasia post-PRK/LASEK is an uncommon event. It occurs in the eyes with altered biomechanics that could be genetically predisposed as well as some known preoperative features that constitute risk factors for

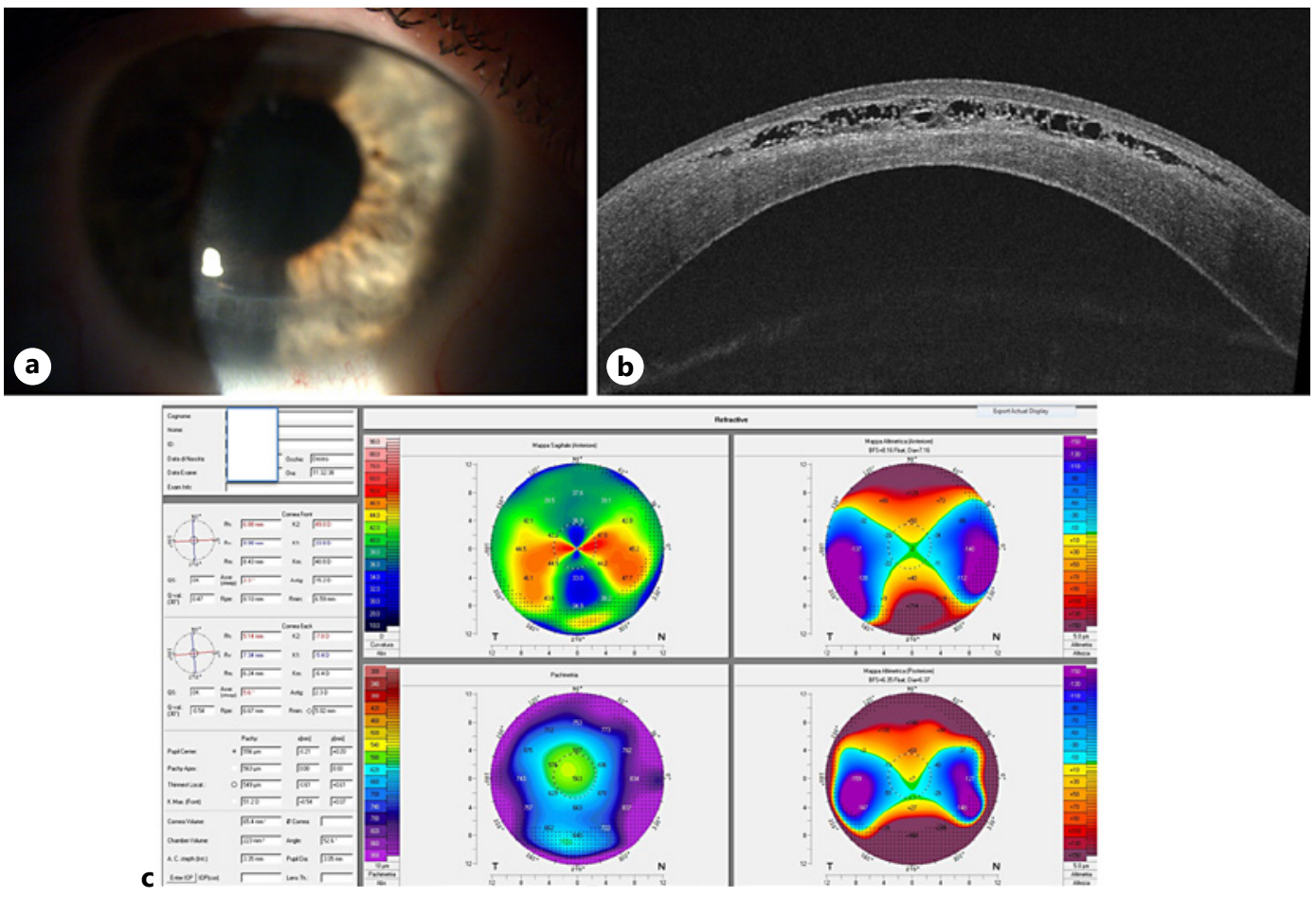

Fig. 2. a Slit-lamp image with inferior lesion extended between 5 and 8 o'clock. b AS-OCT with atypical alterations of the anterior stroma and residual thin tissue. c Corneal tomography showing pellucid-like ectasia with severe alterations of the anterior and posterior elevation. 
ectasia such as asymmetric astigmatism, skewed axis, and thin cornea [4-6]. In the examined patient, we did not detect such risk factors before the laser treatment. Moreover, we believe the changes occurred could not be defined exactly as ectasia even if it resulted in thinning and steepening, but rather an unexpected biomechanical failure with severe unusual stromal changes and tissue disappearance.

Our patient reported a continuous eye rubbing with a mechanical stress with continuous surface damage and incomplete lid closure during sleep. As the eye rubbing was proved to be a risk factor in keratoconus development, we believe that it could contribute also to the corneal alteration onset and their progression in this case [7].

We have not found in the current literature a report matching our case, so we wish to describe this singular corneal complication to share the experience and to enrich the knowledge on complications that might unexpectedly occur after apparently safe excimer laser surface ablation.

\section{Acknowledgements}

This manuscript does not include any nonauthor contributors to acknowledge.

\section{Statement of Ethics}

This research was conducted ethically in accordance with the World Medical Association Declaration of Helsinki and was approved by the Ethical Committee of the University Hospital of Messina (approval number 45/18). The subject has given his written informed consent to publish his case, including publication of images.

\section{Conflict of Interest Statement}

The authors have no conflicts of interest to declare.

\section{Funding Sources}

This study did not receive any funding.

\section{Author Contributions}

Anna M. Roszkowska: study design, drafting the manuscript, and supervision. Giovanni W. Oliverio, Giuseppe A. Signorino, and Mario Urso: data collection and analysis, and drafting the manuscript. Pasquale Aragona: supervision.

\section{Karger'}




\section{References}

1 Seiler T, Quurke AW. Iatrogenic keratectasia after LASIK in a case of forme fruste keratoconus. J Cataract Refract Surg. 1998;24:1007-9.

2 Randleman JB, Russell B, Ward MA, Thompson KP, Stulting RD. Risk factors and prognosis for corneal ectasia after LASIK. Ophthalmology. 2003;110:267-75.

3 Guedj M, Saad A, Audureau E, Gatinel D. Photorefractive keratectomy in patients with suspected keratoconus: five-year follow-up. J Cataract Refract Surg. 2013;39:66-73.

4 Sorkin N, Kaiserman I, Domniz Y, Sela T, Munzer G, Varssano D. Risk assessment for corneal ectasia following photorefractive keratectomy. J Ophthalmol. 2017;2017:2434830.

5 Giri P, Azar DT. Risk profiles of ectasia after keratorefractive surgery. Curr Opin Ophthalmol. 2017;28:337-42.

6 Roszkowska AM, Sommario MS, Urso M, Aragona P. Post photorefractive keratectomy corneal ectasia. Int J Ophthalmol. 2017;10:315-7.

7 Padmanabhan P, Aiswaryah R, Abinaya Priya V. Post-LASIK keratectasia triggered by eye rubbing and treated with topography-guided ablation and collagen cross-linking: a case report. Cornea. 2012;31:575-80. 